\title{
Exploration of the Relationship Between Gut Microbiota and Polycystic Ovary Syndrome (PCOS): a Review
}

\section{Erforschung der Beziehung zwischen intestinaler Mikrobiota und polyzystischem Ovarsyndrom (PCOS): eine Überblicksdarstellung}

\section{(우(1) $(2) \Theta$}

\section{Authors}

Xiaoxuan Zhao ${ }^{1}$, Yuepeng Jiang ${ }^{2}$, Hongyan $\mathrm{Xi}^{1}{ }^{1}$, Lu Chen ${ }^{3}$, Xiaoling Feng ${ }^{3}$

Affiliations

1 Department of Heilongjiang University of Chinese Medicine, Harbin 150040, China

2 Department of Zhejiang Chinese Medical University, Hangzhou 310053, China

3 Department of First Affiliated Hospital of Heilongjiang University of Chinese Medicine, Harbin 150040, China

Key words

polycystic ovary syndrome, gut microbiota, metabolic syndrome, treatment

Schlüsselwörter

polyzystisches Ovarsyndrom, intestinale Mikrobiota, metabolisches Syndrom, Behandlung

received $\quad 16.10 .2019$

revised 10.12.2019

accepted 11.12.2019

Bibliography

DOI https://doi.org/10.1055/a-1081-2036

Geburtsh Frauenheilk 2020; 80: 161-171 @ Georg Thieme

Verlag KG Stuttgart · New York | ISSN 0016-5751

Correspondence

Prof. Xiaoling Feng

First Affiliated Hospital of Heilongjiang University

of Chinese Medicine

Heping, Harbin 150040, Heilongjiang, China

Doctorfxl@163.com

\section{ABSTRACT}

Polycystic ovary syndrome (PCOS) is an endocrine and metabolic syndrome (MS) with a complex etiology, and its pathogenesis is not yet clear. In recent years, the correlation between gut microbiota (GM) and metabolic disease has become a hot topic in research, leading to a number of new ideas about the etiology and pathological mechanisms of PCOS. The literature shows that GM can cause insulin resistance, hyperandrogenism, chronic inflammation and metabolic syndrome (obesity, diabetes) and may contribute to the development of PCOS by influencing energy absorption, the pathways of short chain fatty acids (SCFA), lipopolysaccharides, choline and bile acids, intestinal permeability and the brain-gut axis. As part of the treatment of PCOS, fecal microbiota transplantation, supplementation with prebiotics and traditional Chinese medicine can be used to regulate GM and treat disorders. This article reviews possible mechanisms and treatment options for PCOS, based on methods which target the GM, and offers new ideas for the treatment of PCOS.

\section{ZUSAMMENFASSUNG}

Das polyzystische Ovarsyndrom (PCOS) ist ein endokrines und metabolisches Syndrom (MS) mit einer komplexen Ätiologie. Die Pathogenese von PCOS ist noch immer ungeklärt. In den letzten Jahren hat sich die Forschung verstärkt auf die Interaktion zwischen intestinaler Mikrobiota und Stoffwechselerkrankungen konzentriert, was zu einigen neuen Ideen über die Ätiologie und die pathologischen Mechanismen von PCOS führte. In der Literatur wird berichtet, dass die intestinale Mikroflora Insulinresistenz, Hyperandrogenämie, chronische Entzündungen und metabolisches Syndrom (Adipositas, Diabetes) verursachen kann und durch ihre Auswirkungen auf Energieaufnahme, kurzkettige Fettsäuren, Lipopolysaccharide, Cholin und Gallensäuren, die Permeabilität der Darmschleimhaut sowie die Darm-Hirn-Achse möglicherweise zur Entwicklung von PCOS beitragen kann. Für die Behandlung von PCOS können fäkale Mikrobiomtransfers, eine Supplementierung mit Präbiotika und traditionelle chinesische Medizin zur Regulierung der Darmflora und Behandlung von Störungen eingesetzt werden. Dieser Artikel gibt eine Überblicksdarstellung möglicher Mechanismen und Behandlungsoptionen für PCOS, basierend auf Methoden, die auf eine Änderung der intestinalen Mikrobiota abzielen, und bietet neue Ideen für die Behandlung von PCOS. 


\section{Introduction}

Polycystic ovary syndrome (PCOS) is a common gynecological endocrine disease, with a prevalence of between 8 and 13\% depending on the population studied and the definitions used [1]. The main manifestations are irregular menstrual cycles, infrequent ovulation or anovulation, hyperandrogenemia and polycystic ovarian changes. Several metabolic disorders have been linked to PCOS, including insulin resistance (IR), diabetes and obesity [2,3]. About $50 \%$ of PCOS patients present with varying degrees of IR [4], which in turn serves as a link to other endocrine disorders [5]. In recent years, a new consensus on the diagnosis and treatment of PCOS has emerged, and the idea that metabolic abnormalities and other complications of PCOS should be included in the diagnosis and treatment of PCOS has been emphasized by several reproductive endocrine institutions, including the National Institutes of Health $(\mathrm{NIH})$, the American Endocrine Society, the European Endocrine Society and others $[6,7]$. The diagnosis, treatment and research scope of PCOS has gone beyond reproduction-related fields and now also involves metabolic disease, cardiovascular disease, tumors and other aspects. It is very important to systematically analyze changes to metabolic states when exploring the pathogenesis of PCOS. Modern research shows that GM disorders are closely related to the occurrence and development of metabolic diseases. In the past two decades, some studies have reported a relationship between GM and metabolic syndrome (MS). Larsen et al. [8] studied the changes in the GM composition of patients with type 2 diabetes and found that the quantity of Firmicutes and Clostridium was significantly decreased compared with the control group. Tumbaugh et al. [9] transplanted the GM of obese mice into sterile mice and found that body fat and IR increased significantly after the transplant, while probiotics, such as Bifidobacteria and Lactobacillus, which help to reduce obesity, cholesterol, and weight, declined [10]. These experiments set up a premise which prompted us to investigate the connection between GM and PCOS.

The human microbiome refers to all microorganisms parasitic on the skin and the digestive, respiratory and urogenital tracts, with the gut flora possessing the largest number of microorganisms and most extensive functions [11]. Although GM are only present in the digestive tract, their role goes far beyond regulating the digestive system. A normal GM provides nutrition to the host through energy transport and material exchanges; a delicate balance is established between GM and the host as part of the longterm coexistence process which enables the host to own acquired immunity and establish immune barriers [12]. GM are also widely involved in the storage of fat, the generation of blood vessels, regulation of the nervous system and bone density, and participate in drug metabolism $[13,14]$. Researchers have gradually begun to establish a relationship between GM disorders and the occurrence of diseases. Numerous studies have investigated the relationship between GM disorders and PCOS [15]. Sun et al. [16] discovered that the level of serum dimethylamine was significantly increased in PCOS patients, suggesting that GM activity was higher in the group of women with PCOS. Zhang et al. [17] confirmed that GM were involved in the development of IR and menstrual disorders in PCOS patients by affecting intestinal wall permeability. All the above studies have confirmed the close correlation between GM and PCOS, and this article aims to systematically discuss and summarize the connection between the two and talk about the treatment which targets at the GM, as shown in $>$ Fig. 1, in hope of offering new ideas for the treatment of PCOS. Exploring the specific mechanisms is of great clinical significance.

\section{Overview of GM}

The microecosystem of the human GM is diverse and dynamic. It consists of about 1000-1500 species of bacteria, around 100 trillion bacteria in all, which colonize the human intestinal tract. This figure equates to nearly 10 times the number of human somatic cells, and the number of genes in their genome is 150 times that of their host [18]. Humans inherit roughly 26600 coding genes from their parents, far fewer than other species. This is in sharp contrast to the complexity of humans in terms of their biology, emotions and behavior, also known as the genome-complexity conundrum. Studies have shown that in the human transcriptome, approximately $4 \times 10^{6} \mathrm{mRNAs}$ come from the GM [19], which has also been called "the second genome" [20]. During their longterm symbiosis, the gene complex of human and GM forms and communications at the gene level functionally manifest through extensive participation in various physiological activities, making human physiological activities far more complex than those of other species [21]. Colonization by GM occurs at birth, when babies acquire complex bacteria as they pass through the birth canal. Therefore, the infant GM composition shares obvious maternal characteristics for a time after birth, gradually changing to become more individualized after about one year [22]. When healthy, each person has at least 160 kinds of dominant GM [23]. Although there are individual differences in terms of GM species, the dominant species are basically the same. More than $90 \%$ are Firmicutes and Bacteroides, but typically all humans harbor Bacteroides, Prevotella, Porphyromonas, Clostridium, and Eubacterium. Other less abundantly present bacteria include actinomycetes, proteobacteria and methanogenic archaea [24]. GM are divided into three categories according to their interaction with the host: beneficial bacteria, conditioned pathogens and harmful bacteria. Beneficial bacteria include Lactobacilli and Bifidobacterium, which can inhibit harmful microorganisms, enhance immunity, promote absorption, synthesize vitamins, inhibit tumors, reduce infection, and relieve allergic reactions. Harmful bacteria include Staphylococcus, Salmonella and Campylobacter, which can produce toxins, increase the incidence of cancer and induce infections. Opportunistic pathogens include Enterobacter, Escherichia, and Bacteroides [25]. Under normal circumstances, the bacteria in the GM maintain a delicate dynamic balance to prevent the development of various diseases. A range of diverse factors can lead to changes in GM, including age, diet, and the use of antibiotics. With increasing age, the beneficial bacteria in the intestinal tract decrease, while the toxins produced by harmful bacteria increase, leading to accelerated aging [26]. Diet is one of the most influential factors for GM. Zhang et al. [27] reported on the destruction of GM homeostasis caused by a high-fat diet. It has also been found that abnormal gut motility or immune dysfunction in the intestinal tract and the taking of antibiotics, immunosuppressants or anti-tumor drugs has an adverse effect on GM and may ultimately lead to disease. 


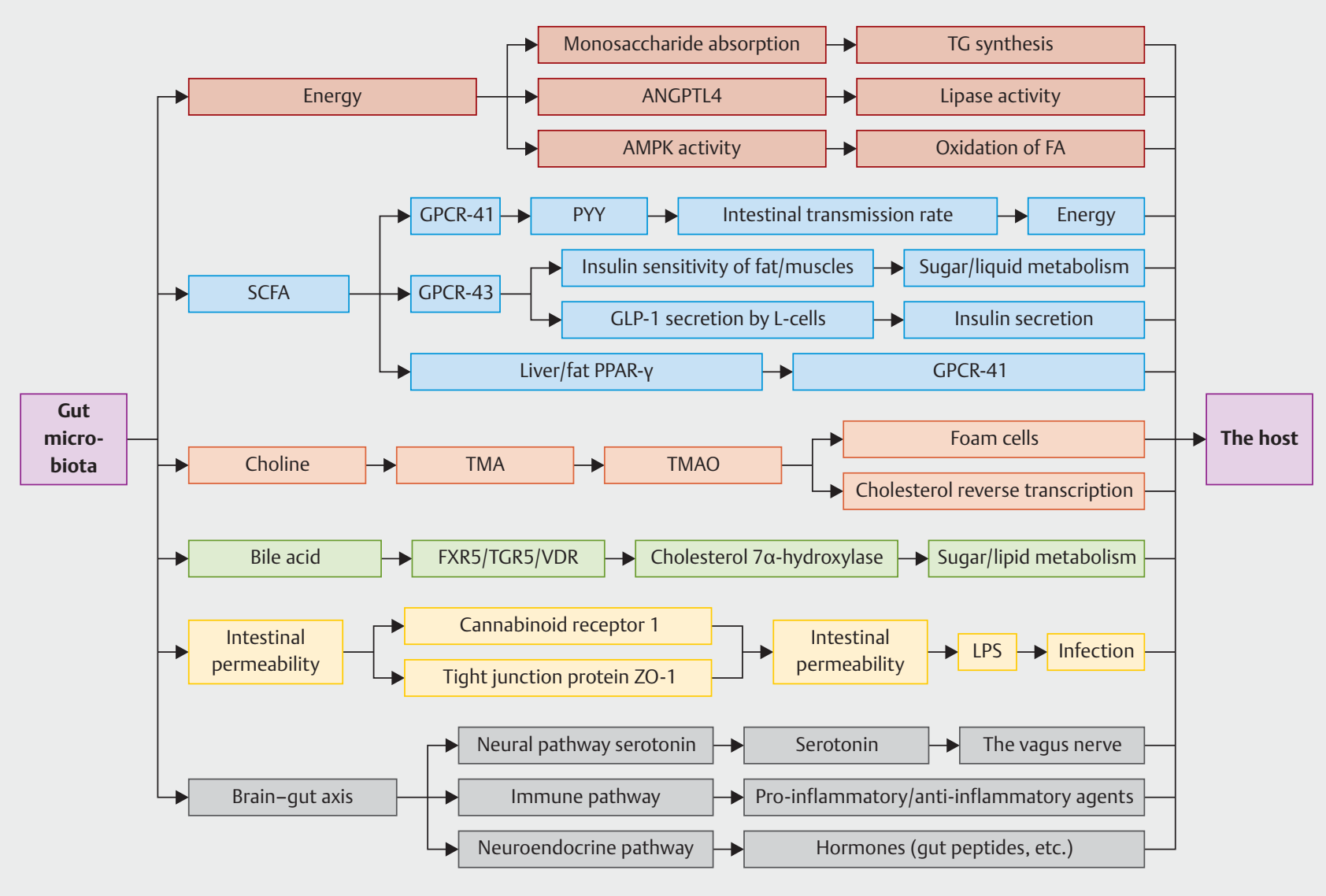

- Fig. 1 Regulation of human health by gut microbiota. TG: triglycerides; ANGPTL4: angiopoietin-like 4; AMPK: 5' adenosine monophosphateactivated protein kinase; FA: fatty acids; ZO-1: zonula occludens-1; LPS: lipopolysaccharides; GPCR-41/43: G-protein-coupled receptor 41/43; PYY: peptide tyrosine tyrosine; GLP-1: glucagon-like peptide-1; PPAR-ү: peroxisome proliferator-activated receptor $\mathrm{Y}$; TMA: trimethylamine; TMAO: trimethylamine oxide; FXR5: farnesoid X receptor 5; VDR: vitamin D receptor; TGR5: G-protein-coupled receptor for bile acids.

\section{Correlation between GM and PCOS}

PCOS is a complex endocrine and metabolic disease. Several PCOS-related genes have been found to be associated with carbohydrate metabolism and the steroid synthesis pathway [28], suggesting an important correlation between metabolic factors and the pathological mechanism of PCOS. GM are involved in various metabolic activities, and accordingly GM are closely associated with the pathogenesis and clinical manifestations of PCOS. Torres et al. [29] studied 73 PCOS patients and found that compared to normal women, patients with PCOS had fewer types of GM, and that this trend was related to an increase in androgens. To study the effect of GM on the host's PCOS phenotype, feces from healthy women and from patients with PCOS were respectively transplanted into two groups of mice by oral lavage. Compared with control mice, the mice transplanted with fecal samples from patients with PCOS developed IR, had an increased number of ovarian cyst-like follicles, a decreased corpus luteum, and higher levels of testosterone and luteinizing hormone. Fertility tests were carried out, in which the number of pups in the first litters after mating were counted. Mice given fecal transplants from women with PCOS produced fewer pups than healthy control mice [30].
Lindheim et al. [31] found that compared with the control group, the relative abundance of Tenericutes ML615J-28 and Bacteroidetes S24-7 was lower in PCOS patients. Another study found that during the construction of a mice model with letrozole-induced PCOS, the total genus of GM decreased but the number of Firmicutes (which are closely related to obesity and MS) increased [32]. From the above experiments, we can infer that GM may be involved in the occurrence and development of PCOS. This could offer a new therapeutic strategy for the clinical diagnosis and treatment of PCOS. Of course, the exact mechanism of the GM in PCOS needs to be supported by further evidence.

\section{The Pathway Mechanism of GM Leading to PCOS}

\section{Energy absorption}

GM degrades carbohydrates into simple sugars and then ferments them into hydrogen, $\mathrm{CO}_{2}, \mathrm{CH}_{4}$, and short chain fatty acids (SCFAs) to provide energy to the host [33]. Obese individuals take more energy from food, which then aggravates the symptoms of obe- 
sity, creating a vicious circle. This phenomenon is associated with a disordered GM. Cani et al. [34] found that a GM disorder in obese patients may increase the host's energy intake by converting substances that cannot be utilized into substances that can be absorbed. SCFAs produced by the fermentation of carbohydrates by the GM can stimulate the release of peptide YY (PYY) in the ileum and colon. PYY inhibits intestinal peristalsis, decreases the secretion of pancreas and promotes the absorption of energy in the intestinal tract $[35,36]$. Turnbaugh et al. [37] analyzed the GM of lean and obese mice with the same diet and found that obese mice had more Firmicutes and fewer Bacteroides than lean mice, and that this distribution of GM was able to absorb energy better. However, after wild aseptic mice were given a GM transplant from obese mice, their energy intake increased and they presented with symptoms of obesity. This study confirmed the relationship between GM, energy metabolism and obesity. Data showed that more than half of PCOS patients were overweight or obese $[38,39]$. Moreover, researchers have confirmed that the richness and phytogenetic diversity of GM species in patients with PCOS is significantly reduced compared with that of control groups [40]. Multiple meta-analyses have confirmed that this is closely related to the obesity phenotype $[41,42]$. To further investigate the relationship between obesity, PCOS and GM, one study compared groups of obese women with PCOS, non-obese women with PCOS, obese women without PCOS and normal controls. The results showed differences in $\beta$ diversity between obese and nonobese women with PCOS [43], but these changes were not observed in non-obese and obese control women. It was speculated that GM may have a specific effect on obesity and energy metabolism in the context of PCOS. From the above experiments, we can conclude that GM disorders can accelerate the process of PCOS by affecting energy absorption.

\section{Short-chain fatty acid (SCFA) metabolism pathway}

Gut microbiota decompose organic materials to produce three major types of SCFAs, including acetate, propionate and butyrate. Den Besten et al. [44] found that SCFAs can activate peroxisome proliferator-activated receptor gamma (PPAR-y) in liver and muscle, thereby regulating glucose uptake and fatty acid oxidation. GM can also affect insulin sensitivity through inflammatory responses mediated by branched-chain amino acids (BCAAs) [45] and SCFAs [46]. Zheng et al. [47] found in three prospective cohort studies that the more BCAAs people ingested, the more likely they were to develop type 2 diabetes [48]. One possible mechanism could be 3-hydroxyisobutyrate (3-HIB), a catabolite of valine, which stimulates the uptake of fatty acids in muscle tissue, leading to fat accumulation and IR [49]. Pedersen et al. [50] revealed the relationship between $\mathrm{GM}$ and BCAA metabolism. It was found that a type of Prevotella in the human intestinal tract was involved in the synthesis of BCAAs. Mice fed a high-fat diet had higher levels of BCAAs in their blood after two weeks, and after three weeks they developed various levels of IR. This animal model exactly simulates the metabolic state of PCOS patients who are obese or who consume a high-fat diet over the long term. SCFAs can combine with free fatty acid receptor-2 (FFAR-2) and FFAR-3 expressed in tissues such as the intestines, fat, skeletal muscle, liver, the immune system and the nervous system to in- hibit the secretion of appetite-stimulating hormone (ASH) by the gastric mucosa [51]. ASH not only inhibits the secretion of $\mathrm{GnRH}$ and of sex hormones [52] but also blocks the transformation of sex hormones. Docanto et al. [53] found that ASH can inhibit the expression of aromatase CYP19A1 in adipo-stromal cells and prevent the transformation of androgen into estrogen. The lower the level of ASH, the higher the androgen level [54]. A meta-analysis indicated that ASH levels of PCOS patients were lower than those of women without PCOS [55]. It would appear that an abnormal SCFA metabolism caused by an abnormal GM is associated with IR and the hyperandrogenemia present in PCOS. High androgen levels in turn can aggravate the abnormal GM, creating a vicious cycle. Kelley et al. [56] found that hyperandrogenemia reduced the diversity of the GM in mice with letrozole-induced PCOS. Similarly, Moreno-Indias et al. [57] found that GM diversity was reduced in female rats exposed to a high-level androgen environment within $24 \mathrm{~h}$ after birth, and that the risk of metabolic diseases in adulthood was increased. Therefore, we draw the conclusion that GM affects the occurrence and development of PCOS through the SCFA pathway.

\section{Lipopolysaccharide metabolic pathway}

Lipopolysaccharides (LPS), also known as bacterial endotoxins, are a unique component in the cell wall of gram-negative bacteria. Bacteroides and Escherichia in the human intestine are gram-negative bacteria [58]. After being absorbed into the blood, LPS can bind to Toll-like receptor 4 (TLR4) on the surface of immune cells through the transmission of LPS-binding protein (LBP), CD14 and bone marrow differentiation factor (MD-2). This can lead to the activation of downstream signaling pathways which promote the expression of TNF- $\alpha$, IL-6, etc. [59], affecting insulin sensitivity and leading to IR. In one study, two groups of mice were fed either a normal or a high-fat diet. After 4 weeks, mice fed a high-fat diet became obese and showed signs of IR; compared with the control group, the concentration of blood LPS in the high-fat diet group was 2-3 times higher, and the GM had changed synchronously. LPS was then subcutaneously injected into wild mice fed a normal diet in this study. After 4 weeks, the control mice had become obese and developed IR [60]. We find that an abnormal GM affects the metabolism, promoting IR and obesity through the LPS pathway. IR is an important clinical characteristic of PCOS [61] and occurs in 50-70\% of patients with PCOS [62]. From the above experiments, we can conclude that the GM mediates IR through LPS. Some researchers believe that IR is the central link in the abnormal metabolism of PCOS and that it promotes a chronic inflammatory state in patients with PCOS. The long-term low release of pro-inflammatory factors can promote hyperandrogenism and obesity in PCOS patients $[63,64]$. Conversely, high androgen levels in PCOS patients also increase inflammation. Su et al. [65] confirmed that androgen activates the ERK $1 / 2 / N F-K B$ signaling pathway and enhances the response of $3 \mathrm{~T} 3-\mathrm{L} 1$ adipocytes to LPS, resulting in the increased secretion of IL-6. Zhu et al. [66] compared the levels of serum LBP in PCOS patients with that of healthy women and found that LBP levels in PCOS patients were significantly increased, irrespective of body mass, providing strong evidence for the involvement of LPS in the pathogenesis of PCOS. 


\section{Choline pathway}

PCOS patients not only suffer from diabetes and obesity, but also have an increased risk of heart disease [67]. A meta-analysis of 104392 subjects showed a significant correlation between PCOS and coronary heart disease (CHD) $(\mathrm{OR}=1.44$; $95 \% \mathrm{Cl}$ : 1.13-1.84; $\mathrm{p}=0.004$ ) [68]. Studies have shown that GM may contribute to the occurrence and development of cardiovascular disease through the choline pathway. Choline is a necessary nutrient for the human body and is mainly obtained from red meat and eggs. It is a component of cell membranes and predominantly involved in lipid metabolism. Choline in food is first fermented to trimethylamine (TMA) by the GM, and then metabolized to trimethylamine oxide (TMAO) by flavin-containing monooxygenase (FMO) in the liver. TMAO is an osmotic substance which plays an important role in maintaining the balance of water and electrolytes both inside and outside cells. TMAO is also involved in regulating cholesterol metabolism, IR, platelet aggregation and inflammatory reactions [10]. Increased TMA-producing bacteria in the intestine can lead to higher TMAO levels, which can result in reverse transport of foam cells and cholesterol, resulting in atherosclerotic lesions and cardiovascular disease [69]. PCOS patients often present with hyperlipidemia and IR [70], which can damage vascular endothelial function and induce thrombosis. These metabolic abnormalities increase the risk of CHD. However, the specific molecular mechanism leading to this pathological state has not yet been explored. Now studies have revealed the mechanism of how GM affects cardiovascular health through the choline pathway. Whether this mechanism plays a role in the occurrence of cardiovascular complications in PCOS patients remains to be verified.

\section{Bile acid pathway}

Bile acids emulsify fats and promote digestion and the absorption of fat-soluble substances in the gastrointestinal tract, while bacteria are essential for the transformation of bile acids. GM regulates bile acid metabolism through farnesoid $X$ receptor (FXR), vitamin $D$ receptor (VDR) and G-protein-coupled receptor for bile acid (TGR5), and participates in the synthesis, metabolism and reabsorption of bile acids. The GM regulates fat synthesis and the level of TG by inhibiting the activity of cholesterol $7 \alpha$-hydroxylase (CYP7A1) [71,72]. Abnormal lipid metabolism is often present in women with PCOS, with a reported incidence of as much as $50 \%$ [73], and it mainly manifests in the form of increased levels of triglycerides (TG), total cholesterol (TC), low-density lipoprotein (LDL) and hormone-sensitive lipase (HSL). Bile acid and decreased concentrations of HDL and Apo A-I [74]. According to the gene and genome analysis of Kyoto Encyclopedia, bile acid metabolism is one of the main metabolic pathways affected by GM in patients with PCOS. Qi et al. [30] conducted an analysis of bile acids in patients with PCOS and normal controls and found that, compared to the control group, glycodeoxycholic acid (GDCA) and tauroursodeoxycholic acid (TUDCA) were significantly decreased in the PCOS group. Correlation analysis showed that Bacteroides forsythus were negatively correlated with GDCA and TUDCA, and interestingly, Bacteroides forsythus were able to uncouple the binding bile acids synthesized in the liver [75]. Compared to the control group, there was a significant increase in the number of bile salt hydrolase genes (encoding bile salt hydrolase enzymes) in PCOS patients. These data suggest that Bacteroides forsythus are significantly expressed in the GM of patients with PCOS, affecting GDCA and TUDCA metabolism in their hosts. In addition, deoxycholic acid induces IL-22 secretion by intestinal lymphocytes via GATA binding protein 3, which in turn improves the PCOS phenotype. This finding is consistent with lower levels of IL-22 in patients with PCOS. In addition, some studies have shown that GM can affect insulin sensitivity through an inflammatory response mediated by bile acids [76]. Based on the above studies, we find that GM affects glucose and lipid metabolism and chronic inflammatory states in PCOS patients by influencing bile acids, leading to other endocrine disorders.

\section{Intestinal permeability}

Intestinal mucosal tight junctions are present at the edge and top of mucosal cell membranes and control the openings of intercellular pathways; they are the first line of defense of the cellular bypass pathway [77]. Intercellular tight junctions are physiologically complete, but under pathological conditions such as oxidative stress or inflammatory damage, their structure and function can be destroyed [78]. Studies have shown that inflammatory factors TNF- $\alpha$ and INF-y can reduce the expression of tight junction proteins zonula occludens-1 (ZO-1) and occludin, leading to a disintegration of tight junctions and increased intestinal permeability [79]. PCOS is considered a chronic inflammatory disease, and the long-term low release of pro-inflammatory factors causes continuous damage to intestinal permeability; this pathological process is closely related to the contribution of the GM. In 2012, Tremellen et al. [80] proposed that one of the pathogenic mechanisms of PCOS could be that obesity and a high-sugar, high-fat diet with low-dietary fiber encourages GM imbalance, thus destroying the junction between intestinal epithelial cells and reducing the expression of ZO-1 and occludin [81]. In addition, the expression of cannabinoid 1 (CD-1) is inhibited, which can also act on tight junction proteins, leading to "leaky gut" [82]. As a result, the process whereby LPS enters the body circulation from gram-negative bacteria accelerates, contributing to an antigen-antibody reaction in vivo which activates the immune system, induces chronic inflammation, hinders the function of insulin receptors, and thereby increases the level of insulin, resulting in high levels of androgens and abnormal follicular development.

\section{Brain-gut axis}

The brain-gut axis is the information-communication system between the intestine and the brain, a neuroendocrine-immune network formed by the central nervous system, the intestinal nervous system (ENS), the hypothalamic-pituitary-adrenal axis and the intestinal tract [83]. GM communicate with the host through the gut-brain axis via immune, neuroendocrine and vagal pathways. Under pathological conditions, GM disorders can result in abnormal secretions of peptides, cytokines and inflammatory factors in the intestinal canal, which transmit information to the brain. After analysis and calculation by the brain, instructions are transmitted to various organs as well as to the intestine and the GM [84,85]. More than 20 of 50 gastrointestinal hormones are known to be involved in the brain-gut interaction [86]. Taking glucagon-like peptide 1 (GLP-1) as an example, studies have shown that GM 
and gastrointestinal hormone secretions in PCOS patients are disordered, with decreased levels of GLP-1 [87], which can affect the gastrointestinal system and the CNS through the vagus nerve; GLP-1 plays a vital role in various functions such as delaying gastric emptying time, reducing appetite, enhancing satiety, promoting the proliferation of pancreatic islet $\beta$ cells and stimulating insulin secretion [88]. Studies have also shown that PCOS patients have common psychological problems such as depression, social phobias, anxiety and aggression [89]. Such psychological conditions are associated with an abnormal brain-gut axis. A particular type of intestinal endocrine cell, enterochromaffin cells, are the major source of the neurotransmitter serotonin $(5-\mathrm{HT})$, with $95 \%$ of the body's 5-HT distributed in the intestine [90]. A GM imbalance causes changes in 5-HT secretion, which affects brain development, stress response, and emotional activities such as anxiety and depression by paracrine [91]. Animal experiments have shown that PCOS mice have significantly lower levels of 5-HT, norepinephrine (NE) and dopamine compared to normal mice.

We have therefore speculated that the emotional disorders exhibited by some PCOS patients may be related to abnormal transmission of information along the brain-gut-GM axis. The literature shows that the intestinal microbiome can also interfere with host immune regulation through the brain-gut axis. Intestinal lymphoid tissues contain $70-80 \%$ of the immune cells of the whole body [92] and provide the anatomical basis for immune regulation. Lang et al. [93] showed that the ratio of T helper cells (Th1/Th2) in the peripheral blood of patients with PCOS-related infertility was higher than that of the control group, and that an imbalance of Th1 and Th2 cells led to poor oocyte quality and ovulation disorders, resulting in lower pregnancy rates for PCOS patients and an increased risk of abortion. Kim et al. [94] found that GM imbalance was significantly correlated with Th1/Th2 ratio, indicating that the balance between GM and helper T cells was both interdependent and mutually restrictive. At present, there is no experimental model which proves that GM regulates immunity through the brain-gut axis, and future research in this area is warranted.

\section{Treatment}

At present, the etiology of PCOS has not been clarified, making it difficult to eradicate the disease. Due to the heterogeneity of patient ages and patient demands and the heterogeneity of the clinical manifestations of PCOS, clinical management must adopt an individualized approach to treat symptoms, based on patients' chief complaints, demands and metabolic changes. Treatment approaches include anti-androgen therapy, insulin sensitizer therapy, or ovulation induction therapy to relieve clinical symptoms, improve fertility, maintain health, and improve patients' quality of life. However, current treatment plans still have many shortcomings of one kind or another, and we need to continue to explore the causes of the disease and find a direct way to treat the disease. Currently, there is no treatment plan which aims to regulate GM, although it may be an effective approach to treat PCOS in future. We have therefore summarized potential treatment options for PCOS based on treating the GM to provide new ideas for clinical treatment.

\section{Lifestyle intervention}

In recent years, numerous studies and guidelines have recommended lifestyle interventions as a first-line treatment for PCOS. This approach focuses on diet, exercise, behavior, psychology and other channels, all of which can affect the activity of the GM and thus regulate the metabolism of the host.

Dietary changes can rapidly change the relative abundance and species in GM [95], something that has been confirmed in patients with MS [96]. A high-fat, high-sugar diet can result in GM disorders, leading to increased intestinal permeability, endotoxemia, hyperandrogenism and ovulation disorders [97]. Therefore, strict dietary controls are needed. Studies have confirmed that the intake of foods with a low glycemic index can make ovulation and menstrual cycles more regular in patients with PCOS [98] and normalize the metabolic index [98].

A recent meta-analysis [99] revealed that exercise can improve the levels of follicle-stimulating hormone, sex hormone-binding globulin, total testosterone and androgen in PCOS patients, and reduce the modified Ferriman-Gallwey (mFG) score. However, the specific mechanism is not clear. In recent years, the more and more attention has focused on the relationship between exercise and GM. Clarke et al. [100] compared the composition of GM in athletes with that of the general population and found that sports can increase GM diversity. Mika et al. [101] studied the effect of exercise on GM in rats and found that, compared to a normally active group, the GM diversity in rats increased after 6 weeks of wheel running. The phylum abundance of Bacteroidetes in exercising rats increased while the phylum abundance of Firmicutes decreased, both of which are significantly correlated with metabolic syndrome. We hypothesize that exercise changes the GM in PCOS patients and thus results in an improvement of metabolic disorders in hosts. Physicians should therefore encourage PCOS patients to do aerobic exercises at $60-80 \%$ of maximum heart rate for more than 30 min at least 4 times per week [102].

Numerous studies have shown that, compared with healthy women, patients with PCOS are more likely to be depressed, anxious and use drugs $[103,104]$. Future research should focus on the relationship between PCOS and mental illness [105]. Studies have shown that cognitive behavioral therapy (CBT) can improve the psychological fatigue of patients with PCOS [106]. Given the bidirectional signaling pathway of the brain-gut axis, we speculated that an improvement in emotional brain activity could affect GM and intestinal homeostasis. However, there are very few studies on the impact of psychological interventions in women with PCOS, and research into the effects of psychological interventions on the GM of patients with PCOS are even rarer. This is one area where we intend to carry out further research.

\section{Fecal microbiota transplantation}

Fecal microbiota transplantation (FMT) is a method whereby microorganisms from the feces of healthy donors are introduced into the small intestine of patients through a nasogastric tube or a nasointestinal tube to directly and rapidly change the composition of the new host's GM and treat disease [107]. Studies suggest that changes to the GM in the colon can lead to Clostridium difficile infections (CDI). FMT has been proposed as a treatment option for $C D I$, and its effectiveness to treat CDI was reported to be more 
than $90 \%$, with few adverse reactions reported [108]. FMT has therefore been recommended in the guidelines as a treatment option for CDI intractable to treatment with antibiotics [109]. FMT can also be used to treat inflammatory bowel disease, chronic constipation and some non-gastrointestinal diseases [110]. Vrieze et al. [111] transplanted the GM of healthy donors into patients with metabolic syndrome, and found that after 6 weeks, the insulin sensitivity of the recipients had increased. At present, there are no clinical reports on the use of FMT to treat gynecological diseases. Guo et al. [112] used FMT in a murine model of PCOS to treat rats. After 36 days, they found significant changes in FMTtreated PCOS rats compared with the untreated control group, with improvements including decreased androgen levels, a significant increase in estradiol and estrone, and normalization of ovarian function. FMT may represent an important new approach to treat PCOS in future, but further study is required.

\section{Probiotic therapy}

Probiotics are defined by the Food and Agricultural Organization (FAO) of the United Nations and the WHO as "live micro-organisms, that (when administered in adequate amounts) confer a health benefit on the host" [113]. Probiotics therapy refers to appropriate supplementation with probiotics to treat disease. Traditional probiotics include Bifidobacterium, Lactobacillus as well as other lactic acid bacteria. Studies have shown that Lactobacillus reuteri GMNL-263 can significantly improve IR, increase satiety, promote intestinal gluconeogenesis, reduce fat accumulation [114] and decrease inflammation in rats with alcoholic fatty liver disease [115]. On a macrogenomic level, it has been found that probiotics can return a GM severely affected by a high-fat diet to its normal state. This suggests that probiotics can be utilized to regulate GM and treat metabolic diseases, which also points to a new therapeutic direction for the treatment of the metabolic abnormalities associated with PCOS [116]. In a randomized, doubleblind, placebo-controlled trial, 60 PCOS patients were randomized into two groups to receive either probiotic capsules $(n=30)$ or placebo $(n=30)$ for 12 weeks. The probiotic capsules contained three viable freeze-dried strains: Lactobacillus acidophilus $\left(2 \times 10^{9} \mathrm{CFU} / \mathrm{g}\right)$, Lactobacillus casei $\left(2 \times 10^{9} \mathrm{CFU} / \mathrm{g}\right)$ and Bifidobacterium bifidum $\left(2 \times 10^{9} \mathrm{CFU} / \mathrm{g}\right)$. Compared with the placebo group, taking probiotic supplements resulted in a significantly reduced BMI ( $-0.2 \pm 0.2$ vs. $\left.+0.03 \pm 0.4 \mathrm{~kg} / \mathrm{m}^{2}, \mathrm{p}=0.004\right)$, lower serum insulin concentrations $(-2.0 \pm 5.8 \mathrm{vs} .+1.6 \pm 5.0 \mu \mathrm{lU} / \mathrm{mL}$, $\mathrm{p}=0.01)$ and lower blood lipid levels $(-13.3 \pm 51.3$ vs. $+13.6 \pm 37.1 \mathrm{mg} / \mathrm{dL}, \mathrm{p}=0.02$ ) [117]. Another randomized double-blind controlled experiment confirmed that compared with the placebo group, probiotic supplementation with Lactobacillus acidophilus, Lactobacillus casei and Bifidobacterium bifidum $\left(2 \times 10^{9} \mathrm{CFU} / \mathrm{g}\right.$ each) significantly increased serum levels of sex hormone-binding globulin (SHBG), reduced total serum testosterone levels, reduced modified Ferriman-Gallwey (mFG) scores, and improved chronic inflammatory states as indicated by decreased levels of serum high-sensitivity C-reactive protein (hs-CRP) and malondialdehyde (MDA) concentrations. No adverse effects of probiotic supplementation were observed [118]. A meta-analysis by Zhang [119] showed that probiotics can reduce total cholesterol, triglycerides and low-density lipoprotein cholesterol levels, in- crease high-density lipoprotein cholesterol and consequently reduce the long-term cardiovascular risk of PCOS patients. But they also pointed out the limitations of current studies on probiotics supplementation, including the fact that the probiotics used in each study varied widely and no standard dosages were used, points which will need to be addressed in future studies.

\section{Prebiotics}

Prebiotics are non-digestible (oligo)saccharides, defined as "selectively fermented ingredients that allow specific changes, both in the composition and/or activity of the gastrointestinal microflora that confers benefits upon host wellbeing and health" [120]. A number of studies have investigated the effects of prebiotics on different metabolic diseases. Some studies have observed that prebiotics can increase microbial fermentation, reduce appetite, and decrease plasma glucose consumption after meals [121]. Urias-Silvas et al. found that adding prebiotics increased the quantity of Bifidobacterium and promoted the secretion of GLP-1 by colon L-cells, thereby improving IR [122]. Inulin, a prebiotic extracted from chicory roots, can improve intestinal microecology and physiological functions and has been shown to increase serum GLP-1 levels, reduce serum IL-6 levels, and normalize the intestinal microecology of diabetic rats [123]. This has led researchers to explore the therapeutic effect of prebiotics on PCOS. Xue et al. [124] divided PCOS model mice into four groups according to an experimental treatment protocol: control group (CON), model group (MOD), inulin group (INU) and metformin group (MET). After 21 days, a significant decrease in body weight and testosterone levels was observed in INU and MET mice; inflammatory markers such as TNF- $\alpha$, IL-6, and IL-17a had decreased, and the PCO status of these mice had improved significantly. GM sequencing showed that Bifidobacteria increased in the INU group compared to the MOD group, while Proteobacteria and Helicobacter decreased. This suggests that inulin improves the metabolic and inflammatory status of PCOS by regulating the gut microflora, similar to the mechanism of metformin. This finding strongly supports the use of prebiotics in the treatment of PCOS.

\section{Traditional Chinese medicine}

Modern research has shown that traditional Chinese medicines contain many ingredients that can improve GM disorders and have a therapeutic effect on PCOS, such as polyphenols, alkaloids, and polysaccharides. Quercetin, a polyphenol, was found to modulate intestinal dysbiosis in rats with high-fat diet-induced obesity by inhibiting the growth of obesity-related bacteria such as Veillonella, Erysipelotrichaceae, Bacillus, and Eubacterium cylindroides [125]. Baicalin can reduce metabolic inflammation by lowering the ratio of gram-negative bacteria to positive bacteria in the intestine, limiting the entry of LPS into the blood and reducing the secretion of inflammatory factors [126]. The main component of Coptis Chinensis is berberine, an alkaloid. It can decrease the number of SCFA-producing bacteria, including Allobaculum, Bacteroides, Blautia, Butyricicoccus, and has a similar therapeutic effect to metformin [127]. Zhang $X$ et al. [128] have proposed that berberine can be used to treat obesity, IR and other metabolic abnormalities by improving GM, and we therefore speculate that one of the mechanisms of berberine in the treatment of PCOS is 
its capacity to regulate GM. Polysaccharides, another group of effective ingredients, can regulate intestinal function through their impact on intestinal immunity, the intestinal barrier, and GM. Polysaccharides from Ophiopogon japonicus were found to increase the proportion of intestinal probiotics in mice fed a highfat diet by increasing the number of Bacteroides and decreasing the number of Firmicutes. MDG-1, a water-soluble $\beta$ - $d$-fructan extracted from the roots of Ophiopogon japonicus, could decrease the ratio of Firmicutes/Bacteroidetes, adjust the abnormal gut microbiota to the normal state and alter their metabolic profiles $[128,129]$. The weight loss effect of astragalus polysaccharide on obese mice whose obesity was induced by a high-fat diet is related to its regulation of GM. A number of polysaccharide compounds have been shown to promote and regulate the growth of intestinal probiotics in mice, including Chinese yam polysaccharide, dogwood polysaccharide, mulberry leaf polysaccharide, and the polysaccharide physalis $[130,131]$. In summary, traditional Chinese medicines, often characterized as multi-component and multi-target compounds, can be used as another therapeutic option to treat PCOS.

\section{Note}

National Natural Science Foundation of China: No.81574014; No. 81904235; NO. bs07

\section{Acknowledgements}

Special thanks are also extended to colleagues at the First Affiliated Hospital of Heilongjiang University of Chinese Medicine and Zhejiang Chinese Medical University.

Conflict of Interest

The authors declare that they have no conflict of interest.

\section{References}

[1] Azziz R, Carmina E, Chen Z et al. Polycystic ovary syndrome. Nat Rev Dis Primers 2016; 2: 16057

[2] Li R, Zhang Q, Yang D et al. Prevalence of polycystic ovary syndrome in women in China: a large community-based study. Hum Reprod 2013; 28: 2562-2569

[3] Teede HJ, Misso ML, Costello MF et al. Recommendations from the international evidence-based guideline for the assessment and management of polycystic ovary syndrome [J]. Hum Reprod 2019; 34: 388. doi:10.1093/humrep/dey363

[4] Luque-Ramírez M, Escobar-Morreale HF. Polycystic ovary syndrome as a paradigm for prehypertension, prediabetes, and preobesity. Curr Hypertens Rep 2014; 16: 500

[5] Randeva HS, Tan BK, Weickert MO et al. Cardiometabolic aspects of the polycystic ovary syndrome. Endocr Rev 2012; 33: 812-841

[6] Johnson T, Kaplan L, Ouyang P et al. National Institutes of Health evidence-based Methodology Workshop on polycystic Ovary Syndrome (PCOS). NIH Eb MW report[C]. Bethesda: National Institutes of Health; 2012

[7] Legro RS, Arslanian SA, Ehrmann DA et al. Diagnosis and treatment of polycystic ovary syndrome: an endocrine society clinical practice guideline. J Clin Endocrinol Metab 2013; 98: 4565-4592
[8] He Q, Li X, Liu C et al. Dysbiosis of the fecal microbiota in the TNBS-induced Crohn's disease mouse model. Appl Microbiol Biotechnol 2016; 100: $4485-4494$

[9] Turnbaugh P], Backhed F, Fulton L et al. Diet-induced obesity is linked to marked but reversible alterations in the mouse distal gut microbiome. Cell Host Microbe 2008; 3: 213-223

[10] Chen J, Wang R, Li XF et al. Bifidobacterium adolescentis supplementation ameliorates visceral fat accumulation and insulin sensitivity in an experimental model of the metabolic syndrome. $\mathrm{Br}$ J Nutr 2012; 107 : 1429-1434. doi:10.1017/S0007114511004491

[11] Coyte KZ, Schluter J, Foster KR. The ecology of the microbiome: networks, competition, and stability. Science 2015; 350: 663-666

[12] Pickard JM, Chervonsky AV. Intestinal fucose as a mediator of host-microbe symbiosis. J Immunol 2015; 194: 5588-5593

[13] Macfarlane GT, Macfarlane S. Human colonic microbiota: ecology, physiology and metabolic potential of intestinal bacteria. Scand J Gastroenterol Suppl. 1997; 222: 3-9

[14] Consortium HMP. Structure, function and diversity of the healthy human microbiome. Nature 2012; 486: 207-214

[15] Kant R, de Vos WM, Palva A et al. Immunostimulatory CpG motifs in the genomes of gut bacteria and their role in human health and disease. J Med Microbiol 2014; 63 (Pt 2): 293

[16] Sun L, Hu W, Liu Q et al. Metabonomics Reveals Plasma Metabolic Changes and Inflammatory Marker in Polycystic Ovary Syndrome Patients. J Proteome Res 2012; 11: 2937-2946

[17] Zhang D, Zhang L, Yue F et al. Serum zonulin is elevated in women with polycystic ovary syndrome and correlates with insulin resistance and severity of anovulation. Eur J Endocrinol 2015; 172: 29-36

[18] Gill SR, Pop M, De Boy RT et al. Metagenomic analysis of the human distal gut microbiome. Science 2006; 312: 1355-1359

[19] Ouyang S, Liu J, Jones KM et al. The map-based sequence of the rice genome. Nature 2005; 436: 793-800

[20] Human Microbiome Project Consortium. A framework for human microbiome research OPEN [J]. Nature 2012; 486: 215-221. doi:10.1038/ nature 11209

[21] Hellman AB. Gut bacteria gene complement dwarfs human genome [J]. Nature 2010; 464: 59-65

[22] Grenham S, Clarke G, Cryan JF et al. Brain-gut-microbe communication in health and disease. Front Physiol 2011; 2: 94

[23] Faith JJ, Guruge JL, Charbonneau M et al. The long-term stability of the human gut microbiota. Science 2013; 341: 1237439

[24] Lozupone CA, Stombaugh JI, Gordon Jl et al. Diversity, stability and resilience of the human gut microbiota. Nature 2012; 489: 220-230

[25] Clemente JC, Ursell LK, Parfrey LW et al. The impact of the gut microbiota on human health: an integrative view. Cell 2012; 148: 1258-1270

[26] Vaiserman AM, Koliada AK, Marotta F. Gut microbiota: a player in aging and a target for anti-aging intervention. Ageing Res Rev 2017; 35: 36-45

[27] Zhang C, Zhang M, Pang $X$ et al. Structural resilience of the gut microbiota in adult mice under high-fat dietary perturbations. ISME J 2012; 6: 1848-1857

[28] Li T, Wu K, You L et al. Common variant rs9939609 in gene FTO confers risk to polycystic ovary syndrome. PLoS One 2013; 8: e66250

[29] Torres PJ, Siakowska M, Banaszewska B et al. Gut microbial diversity in women with polycystic ovary syndrome correlates with hyperandrogenism. J Clin Endocrinol Metab 2018; 103: 1502-1511

[30] Qi X, Yun C, Sun L et al. Gut microbiota-bile acid-interleukin-22 axis orchestrates polycystic ovary syndrome [J]. Nat Med 2019; 25: 1225-1233

[31] Lindheim L, Bashir M, Münzker J et al. Alterations in gut microbiome composition and barrier function are associated with reproductive and metabolic defects in women with polycystic ovary syndrome (PCOS): a pilot study. PLoS One 2017; 12: e0168390 
[32] Kelley ST, Skarra DV, Rivera A] et al. The gut microbiome is altered in a letrozole-induced mouse model of polycystic ovary syndrome. PLoS One 2016; 11: e0146509

[33] Everard A, Cani PD. Gut microbiota and GLP-1. Rev Endocr Metab Disord 2014; 15: 189-196

[34] Cani PD, Delzenne NM, Amar J et al. Role of gut microflora in the development of obesity and insulin resistance following high-fat diet feeding [J]. Pathologie Biologie 2008: 56: 305-309

[35] Karaki S, Mitsui R, Hayashi $H$ et al. Short-chain fatty acid receptor, GPR43, is expressed by enteroendocrine cells and mucosal mast cells in rat intestine [J]. Cell Tissue Res 2006; 324: 353-360

[36] Karra E, Chandarana K, Batterharm RL. The role of peptide YY in appetite regulation and obesity. J Physiol 2009; 587 (Pt 1): 19-25

[37] Turnbaugh PJ, Ley RE, Mahowald MA et al. An obesity-associated gut microbiome with increased capacity for energy harvest. Nature 2006; 444: 1027-1031

[38] Lindheim L, Bashir M, Münzker J et al. Alterations in Gut Microbiome Composition and Barrier Function Are Associated with Reproductive and Metabolic Defects in Women with Polycystic Ovary Syndrome (PCOS): A Pilot Study. PloS One 2017; 12: e0168390

[39] Liu R, Zhang C, Shi Y et al. Dysbiosis of gut microbiota associated with clinical parameters in polycystic ovary syndrome [J]. Front Microbiol 2017; 8: 324. doi:10.3389/fmicb.2017.00324. eCollection 2017

[40] Torres PJ, Siakowska M, Banaszewska B et al. Gut microbial diversity in women with polycystic ovary syndrome correlates with hyperandrogenism [J]. J Clin Endocrinol Metab 2018; 103: 1502-1511. doi:10.1210/ jc.2017-02153

[41] Walters WA, Xu Z, Knight R. Meta-analyses of human gut microbes associated with obesity and IBD. FEBS Lett 2014; 588: 4223-4233

[42] Tilman D. Diversity and Productivity in a Long-Term Grassland Experiment. Science 2001; 294: 843-845

[43] Insenser M, Murri M, Del Campo R et al. Gut microbiota and the polycystic ovary syndrome: influence of sex, sex hormones, and obesity. J Clin Endocrinol Metab 2018; 103: 2552-2562. doi:10.1210/jc.201702799

[44] den Besten G, Lange K, Havinga R et al. Gut-derived short-chain fatty acids are vividly assimilated into host carbohydrates and lipids. Am J Physiol Gastrointest Liver Physiol 2013; 305: G900-G910

[45] Saad M], Santos A, Prada PO. Linking gut microbiota and inflammation to obesity and insulin resistance. Physiology (Bethesda) 2016; 31: 283293

[46] Mollica MP, Mattace RG, Cavaliere G et al. Butyrate regulates liver mitochondrial function, efficiency, and dynamics in insulin-resistant obese mice. Diabetes 2017; 66: 1405-1418

[47] Zheng Y, Li Y, Qi Q et al. Cumulative consumption of branched-chain amino acids and incidence of type 2 diabetes. Int J Epidemiol 2016; 45: 1482-1492

[48] Lotta LA, Scott RA, Sharp SJ et al. Genetic predisposition to an impaired metabolism of the branched-chain amino acids and risk of type 2 diabetes: a mendelian randomisation analysis. PLoS Med 2016; 13: e1002179

[49] Neis EP, Dejong CH, Rensen SS. The role of microbial amino acid metabolism in host metabolism. Nutrients 2015; 7: 2930-2946

[50] Pedersen HK, Gudmundsdottir V, Nielsen HB et al. Human gut microbes impact host serum metabolome and insulin sensitivity [J]. Nature 2016; 535: 376-381

[51] Engelstoft MS, Schwartz TW. Opposite regulation of ghrelin and glucagon-like peptide- 1 by metabolite g-protein-coupled receptors. Trends Endocrinol Metab 2016; 27: 665-675

[52] Koh A, De Vadder F, Kovatcheva-Datchary P et al. From dietary fiber to host physiology: short-chain fatty acids as key bacterial metabolites. Cell 2016; 165: 1332-1345
[53] Au CMC, Docanto MM, Zahid H et al. Des-acyl ghrelin inhibits the capacity of macrophages to stimulate the expression of aromatase in breast adipose stromal cells [J]. J Steroid Biochem Mol Biol 2017; 170: 49-53

[54] Novelle MG, Vázquez M], Martinello KD et al. Neonatal events, such as androgenization and postnatal overfeeding, modify the response to ghrelin [J]. Sci Rep 2014; 4: 4855

[55] Gao T, Wu L, Chang F et al. Low circulating ghrelin levels in women with polycystic ovary syndrome: a systematic review and meta-analysis. Endocr J 2016; 63: 93-100

[56] Kelley ST, Skarra DV, Rivera A] et al. The gut microbiome is altered in a letrozole-induced mouse model of polycystic ovary syndrome. PLoS One 2016; 11: e0146509

[57] Moreno-Indias I, Sanchez-Alcoholado L, Sanchez-Garrido MA et al. Neonatal androgen exposure causes persistent gut microbiota dysbiosis related to metabolic disease in adult female rats. Endocrinology 2016; 157: 4888-4898

[58] González F. Inflammation in polycystic ovary syndrome: underpinning of insulin resistance and ovarian dysfunction. Steroids 2012; 77: 300-305

[59] Chassaing B, Ley RE, Gewirtz AT. Intestinal epithelial cell toll-like receptor 5 regulates the intestinal microbiota to prevent low-grade inflammation and metabolic syndrome in mice. Gastroenterology 2014; 147: 13631377.e17

[60] Cani PD, Amar J, Iglesias MA et al. Metabolic endotoxemia initiates obesity and insulin resistance. Diabetes 2007; 56: 1761-1772

[61] Fruzzetti F, Perini D, Russo M et al. Comparison of two insulin sensitizers, metformin and myo-inositol, in women with polycystic ovary syndrome (PCOS). Gynecol Endocrinol 2016; 33: 1-4

[62] Polak K, Czyzyk A, Simoncini T et al. New markers of insulin resistance in polycystic ovary syndrome []]. J Endocrinol Invest 2017; 40: 1-8

[63] Belani M, Deo A, Shah P et al. Differential insulin and steroidogenic signaling in insulin resistant and non- insulin resistant human luteinized granulosa cells - a study in PCOS patients. I Steroid Biochem Mol Biol 2018; 178: 283-292

[64] Glintborg D, Petersen MH, Ravn P et al. Comparison of regional fat mass measurement by whole body DXA scans and anthropometric measures to predict insulin resistance in women with polycystic ovary syndrome and controls [J]. Acta Obstet Gynecol Scand 2016; 95: 1235-1243

[65] Su C, Chen M, Huang $\mathrm{H}$ et al. Testosterone enhances lipopolysaccharideinduced interleukin- 6 and macrophage chemotactic protein- 1 expression by activating the extracellular signal-regulated kinase $1 / 2$ /nuclear factor-KB signalling pathways in 3T3-L1 adipocytes. Mol Med Rep 2015; 12: 696-704

[66] Zhu Q, Zhou H, Zhang A et al. Serum LBP is associated with insulin resistance in women with PCOS. PLoS One 2016; 11: e0145337

[67] Ali AT. Polycystic ovary syndrome and metabolic syndrome. Ceska Gynekol 2015; 80: 279

[68] Zhao L, Zhu Z, Lou H et al. Polycystic ovary syndrome (PCOS) and the risk of coronary heart disease (CHD): a meta-analysis [J]. Oncotarget 2016; 7: 33715

[69] Koeth RA, Wang Z, Levison BS et al. Intestinal microbiota metabolism of L-carnitine, a nutrient in red meat, promotes atherosclerosis. Nat Med 2013; 19: 576-585

[70] Bickerton AST, Clark N, Meeking D et al. Cardiovascular risk in women with polycystic ovarian syndrome (PCOS) [J]. J Clin Pathol 2005; 58 : 151-154

[71] Sayin SI, Wahlström A, Felin J et al. Gut microbiota regulates bile acid metabolism by reducing the levels of tauro-beta-muricholic acid, a naturally occurring FXR antagonist. Cell Metab 2013; 17: 225-235

[72] Zhang J, Fan P, Liu H et al. Apolipoprotein A-I and B levels, dyslipidemia and metabolic syndrome in south-west Chinese women with PCOS. Hum Reprod 2012; 27: 2484-2493 
[73] Cullberg G, Hamberger L, Mattsson L et al. Lipid metabolic studies in women with a polycystic ovary syndrome during treatment with a lowdose desogestrel-ethinylestradiol combination. Acta Obstet Gynecol Scand 2011; 64: 203-207

[74] Li S, Chu Q, Ma J et al. Discovery of novel lipid profiles in PCOS: Do insulin and androgen oppositely regulate bioactive lipid production?. J Clin Endocrinol Metab 2017; 102: 810-821

[75] Shindo K, Machida M, Fukumura $M$ et al. Omeprazole induces altered bile acid metabolism. Gut 1998; 42: 266-271

[76] Fujisaka S, Ussar S, Clish C et al. Antibiotic effects on gut microbiota and metabolism are host dependent. J Clin Invest 2016; 126: 4430-4443

[77] Terry S, Nie M, Matter $\mathrm{K}$ et al. Rho signaling and tight junction functions. Physiology (Bethesda) 2010; 25: 16-26

[78] Assimakopoulos SF, Tsamandas AC, Louvros E et al. Intestinal epithelial cell proliferation, apoptosis and expression of tight junction proteins in patients with obstructive jaundice. Eur J Clin Invest 2011; 41: 117-125

[79] Patrick DM, Leone AK, Shellenberger J] et al. Proinfl ammatory cytokines tumor necrosis factor-alpha and interferon-gamma modulate epithelial barrier function in Madin-Darby canine kidney cells through mitogen activated protein kinase signaling. BMC Physiol 2006; 6: 2

[80] Tremellen K, Pearce K. Dysbiosis of gut microbiota (DOGMA): a novel theory for the development of polycystic ovarian syndrome. Med Hypotheses 2012; 79: 104-112

[81] Lam YY, Ha CW, Campbell CR et al. Increased gut permeability and microbiota change associate with mesenteric fat inflammation and metabolic dysfunction in diet-induced obese mice. PLoS One 2012; 7: e34233

[82] Muccioli GG, Naslain D, Backhed F et al. The endocannabinoid system links gut microbiota to adipogenesis. Mol Syst Biol 2010; 6: 392

[83] Mayer EA. Gut feelings: the emerging biology of gut-brain communication. Nat Rev Neurosci 2011; 12: 453-466

[84] Katherine GC, Iris G, Montserrat P et al. Effects of flavonoids on intestinal inflammation, barrier integrity and changes in gut microbiota during diet-induced obesity. Nutr Res Rev 2016; 29: 234-248

[85] Dong CZ, Choi S, Shahi PK et al. Inhibition of pacemaker activity in interstitial cells of Cajal by LPS via NF-KB and MAP kinase. World J Gastroenterol 2013; 19: 1210-1218

[86] Lach G, Schellekens H, Dinan TG et al. Anxiety, Depression, and the Microbiome: A Role for Gut Peptides. Neurotherapeutics 2017; 15: 1-24

[87] Aydin K, Arusoglu G, Koksal G et al. Fasting and post-prandialglucagon like peptide 1 and oral contraception in polycystic ovary syndrome. Clin Endocrinol (Oxf) 2014; 81: 588-592

[88] Sun L, Ji C, Jin L et al. Effects of Exenatide on Metabolic Changes, Sexual Hormones, Inflammatory Cytokines, Adipokines, and Weight Change in a DHEA-Treated Rat Model. Reprod Sci 2016; 23: 1242-1249

[89] Kohlné Papp I. Psychosocial approach of polycystic ovary syndrome. Orv Hetil 2014; 155: 1867-1871

[90] Gershon MD, Tack J. The serotonin signaling system: From basic understanding to drug development for functional GI disorders. Gastroenterology 2007; 132: 397-414

[91] Chaudhari NK, Nampoothiri LP. Neurotransmitter alteration in a testosterone propionate- induced polycystic ovarian syndrome rat model. Horm Mol Biol Clin Investig 2017; 29: 71-77

[92] Riaz T, Sollid LM, Olsen I et al. Quantitative Proteomics of Gut-Derived Th1 and Th1/Th17 Clones Reveal the Presence of CD28+ NKG2D- Th1 Cytotoxic CD4+ T cells. Mol Cell Proteomics 2016; 15: 1007-1016

[93] Lang Q, Xu W, Li X et al. Differential expression profile of immunological cytokines in local ovary in patients with polycystic ovarian syndrome: analysis by flow cytometry. Eur J Obstet Gynecol 2016; 197: 136-141

[94] Kim CH, Jeongho P, Myunghoo K. Gut Microbiota-Derived Short-Chain Fatty Acids, T Cells, and Inflammation. Immune Netw 2014; 14: 277282
[95] Turnbaugh PJ, Ridaura VK, Faith |] et al. The effect of diet on the human gut microbiome: a metagenomic analysis in humanized gnotobiotic mice. Sci Transl Med 2009; 1: 6799-6806

[96] Fava F, Gitau R, Griffin BA et al. The type and quantity of dietary fat and carbohydrate alter faecal microbiome and short-chain fatty acid excretion in a metabolic syndrome 'at-risk' population. Int J Obes (Lond) 2013; 37: 216-223

[97] Jakubowicz D, Barnea M, Wainstein J et al. Effects of caloric intake timing on insulin resistance and hyperandrogenism in lean women with polycystic ovary syndrome. Clin Sci (Lond) 2013; 125: 423-432

[98] Moran LJ, Ko H, Misso M et al. Dietary composition in the treatment of polycystic ovary syndrome: a systematic review to inform evidencebased guidelines [J]. J Acad Nutr Diet 2013; 113: 520-545

[99] Haqq L, Mc Farlane J, Dieberg G et al. Effect of lifestyle intervention on the reproductive endocrine profile in women with polycystic ovarian syndrome: a systematic review and meta-analysis. Endocr Connect 2013; 3: 36-46

[100] Clarke SF, Murphy EF, O’Sullivan O et al. Exercise and associated dietary extremes impact on gut microbial diversity. Gut 2014; 63: 1913-1920

[101] Mika A, Van Treuren W, González A et al. Exercise is more effective at altering gut microbial composition and producing stable changes in lean mass in juvenile versus adult male F344 rats. PLoS One 2015; 10 : e125889

[102] Harrison CL, Lombard CB, Moran LJ et al. Exercise therapy in polycystic ovary syndrome: a systematic review. Hum Reprod Update 2011; 17 : $171-183$

[103] Hart R. Polycystic ovarian syndrome-prognosis and treatment outcomes. Curr Opin Obstet Gynecol 2007; 19: 529-535

[104] Teede HJ, Misso ML, Deeks AA et al. Assessment and management of polycystic ovary syndrome: summary of an evidence-based guideline. Med J Aust 2011; 195: S65

[105] El Hayek S, Bitar L, Hamdar LH et al. Poly cystic ovarian syndrome: an updated overview [I]. Front Physiol 2016; 7: 124

[106] Abdollahi L, Mirghafourvand M, Babapour JK et al. Effectiveness of cognitive-behavioral therapy (CBT) in improving the quality of life and psychological fatigue in women with polycystic ovarian syndrome: a randomized controlled clinical trial [J]. J Psychosom Obstet Gynaecol 2019; 40: 283-293

[107] Moore T, Rodriguez A, Bakken JS. Fecal microbiota transplantation. J Neurogastroenterol Motil 2015; 21: 294-295

[108] Kociolek LK, Gerding DN. Breakthroughs in the treatment and prevention of Clostridium difficile infection. Nat Rev Gastroenterol Hepatol 2016; 13: 150-160

[109] van Beurden YH, de Groot PF, van Nood E et al. Complications, effectiveness, and long term follow-up of fecal microbiota transfer by nasoduodenal tube for treatment of recurrent Clostridium difficile infection. United European Gastroenterol ] 2017; 5: 868-879

[110] Panchal P, Budree S, Scheeler A et al. Scaling safe access to fecal microbiota transplantation: past, present, and future. Curr Gastroenterol Rep 2018; 20: 28

[111] Vrieze A, Van Nood E, Holleman F et al. Transfer of intestinal microbiota from lean donors increases insulin sensitivity in individuals with metabolic syndrome. Gastroenterology 2012; 143: 913-916

[112] Guo Y, Qi Y, Yang X et al. Association between Polycystic Ovary Syndrome and Gut Microbiota [J]. PLoS One 2016; 11: e0153196. doi:10.1371/journal.pone.0153196. eCollection 2016

[113] Hotel ACP, Cordoba A. Health and nutritional properties of probiotics in food including powder milk with live lactic acid bacteria [J]. Prevention 2001; 5: 1-10

[114] Hsieh FC, Lee CL, Chai CY et al. Oral administration of Lactobacillus reuteri GMNL-263 improves insulin resistance and ameliorates hepatic steatosis in high fructose-fed rats. Nutr Metab 2013; 10: 35 
[115] Liang Y], Lin CL, Zhang YP et al. Probiotic mixture of Lactobacillus and Bifdobacterium alleviates systemic adiposity and infammation in nonalcoholic fatty liver disease rats through Gpr109a and the commensal metabolite butyrate. Inflammopharmacology 2018; 26: 1051-1055

[116] Bäckhed F, Ding H, Wang T et al. The gut microbiota as an environmental factor that regulates fat storage. Proc Natl Acad Sci 2004; 101: 15718-15723

[117] Ahmadi S, Jamilian M, Karamali M et al. Probiotic supplementation and the effects on weight loss, glycaemia and lipid profiles in women with polycystic ovary syndrome: a randomized, double-blind, placebo-controlled trial [J]. Hum Fertil (Camb) 2017; 20: 254-261

[118] Karamali M, Eghbalpour S, Rajabi S et al. Effects of Probiotic Supplementation on Hormonal Profiles, Biomarkers of Inflammation and Oxidative Stress in Women With Polycystic Ovary Syndrome: A Randomized, Double-Blind, Placebo-Controlled Trial. Arch Iran Med 2018; 21: $1-7$

[119] Zhang H, Wen W, Shen J et al. Effect of microecological preparation supplementation on woman with polycystic ovary syndrome: A metaanalysis protocol [J]. Medicine (Baltimore) 2018; 97: e13040

[120] Gibson GR, Scott KP, Rastall RA et al. Dietary prebiotics: current status and new definition. Food Sci Technol Bull Funct Foods 2010; 7: 1-19

[121] Nilsson AC, Ostman EM, Holst J] et al. Including indigestible carbohydrates in the evening meal of healthy subjects improves glucose tolerance, lowers inflammatory markers, and increases satiety after a subsequent standardized breakfast. J Nutr 2008; 138: 732-739

[122] Urías-Silvas JE, Cani PD, Delmée E et al. Physiological effects of dietary fructans extracted from Agave tequilana Gto and Dasylirion spp. $\mathrm{Br}$ J Nutr 2008; 99: 254-261
[123] Zhang Q, Yu H, Xiao X et al. Inulin-type fructan improves diabetic phenotype and gut microbiota profiles in rats [J]. Peer] 2018; 6: e4446

[124] Xue J, Li X, Liu P et al. Inulin and metformin ameliorate polycystic ovary syndrome via anti-inflammation and modulating gut microbiota in mice [J]. Endocr ] 2019; 66: 859-870

[125] Etxeberria U, Arias N, Boqué N et al. Reshaping faecal gut microbiota composition by the intake of trans-resveratrol and quercetin in highfat sucrose diet-fed rats. J Nutr Biochem 2015; 26: 651

[126] Chen H, Xu Y, Wang J et al. Baicalin ameliorates isoproterenol-induced acute myocardial infarction through iNOS, inflammation and oxidative stress in rat. Int J Clin Exp Pathol 2015; 8: 10139-10147

[127] Wang K, Feng X, Chai L et al. The metabolism of berberine and its contribution to the pharmacological effects [J]. Drug Metab Rev 2017; 49: 139-157

[128] Zhang X, Zhao Y, Xu J et al. Modulation of gut microbiota byberberine and metformin during the treatment of high-fat diet-induced obesity in rats. Sci Rep 2015; 5: 14405

[129] Shi LL, Li Y, Wang Y et al. MDG-1, an Ophiopogon polysaccharide, regulate gut microbiota in high-fat diet-induced obese C57BL/6 mice [J]. Int J Biol Macromol 2015; 81: 576-583. doi:10.1016/j.ijbiomac.2015.08.057

[130] Ming L, Yue-Fei W, Guan-Wei F et al. Balancing Herbal Medicine and Functional Food for Prevention and Treatment of Cardiometabolic Diseases through Modulating Gut Microbiota [J]. Frontiers in Microbiology 2017; 8: 2146

[131] Chang C], Lin CS, Lu CC et al. Ganoderma lucidum reduces obesity in mice by modulating the composition of the gut microbiota [J]. Nature Communications 2015; 6: 7489 